\title{
Optimal design of vertically emitting circular Bragg disk resonator lasers
}

\section{Xiankai Sun, Jacob Scheuer, Amnon Yariv}

Xiankai Sun, Jacob Scheuer, Amnon Yariv, "Optimal design of vertically emitting circular Bragg disk resonator lasers," Proc. SPIE 6896, Integrated Optics: Devices, Materials, and Technologies XII, 689604 (12 February 2008); doi: $10.1117 / 12.763948$

Event: Integrated Optoelectronic Devices 2008, 2008, San Jose, California, United States 


\title{
Optimal design of vertically emitting circular Bragg disk resonator lasers
}

\author{
Xiankai Sun*a, Jacob Scheuer ${ }^{\mathrm{b}}$, Amnon Yariv $^{\mathrm{a}}$ \\ ${ }^{a}$ Dept. of Applied Physics, California Institute of Technology, Pasadena, CA, USA 91125; \\ ${ }^{\mathrm{b}} \mathrm{School}$ of Electrical Engineering, Tel-Aviv University, Ramat-Aviv, Tel-Aviv, Israel 69978
}

\begin{abstract}
We derive a comprehensive coupled-mode theory, including resonant vertical radiation, for the analysis of non-periodic grating circular Bragg lasers. We analyze the threshold levels and modal properties of such lasers employing mixedorder Bragg gratings to achieve both strong confinement and efficient vertical emission. By reducing the threshold gain and maximizing the emission efficiency, we suggest an optimal design for the circular Bragg microdisk lasers which indicates low-threshold and high-efficiency operation is possible.
\end{abstract}

Keywords: Bragg reflectors, disk resonators, vertical emitting lasers, semiconductor lasers, integrated optics, coupledmode theory, optimal design

\section{INTRODUCTION}

Circular optical resonators have found their applications in optical communication systems, cavity quantum electrodynamics (QED), and biochemical sensing. In many applications, both a high quality factor $(Q)$ and a small modal volume are desirable. It's hard to achieve both high $Q$ and small modal volume in conventional circular resonators employing the total internal reflection mechanism for light confinement, since small modal volume requires small bending radius, which results in large bending loss and a decreased $Q$. This linkage can be broken by using Bragg reflection. The "Annular Bragg Resonators (ABRs)," which are designed to confine the light in a central disk or a circumferentially guiding defect by radial Bragg reflection, can provide a complete 2-D feedback and strongly localize the light in the predesigned region ${ }^{1,2}$.

The properties of passive ABRs have been theoretically studied, yielding optimal Bragg layer structures ${ }^{1}$. Since we have experimentally realized ABR based lasers in InGaAsP gain medium ${ }^{3}$, a comprehensive theoretical analysis of active ABRs (i.e., lasers) and optimization of their threshold level and efficiency are necessary. So in this work, we derive a comprehensive coupled-mode theory, including resonant vertical radiation, for the analysis of non-periodic circular Bragg lasers. Then we apply the theory to a disk resonator configuration and obtain the governing characteristic equation. From the characteristic equation, we numerically analyze the threshold gain levels and modal fields for such lasers. Since such lasers, if optimally designed, can have both strong confinement and efficient vertical emission, we suggest a design procedure, by which low-threshold operation and high-efficiency emission can be satisfied at the same time.

\section{COMPREHENSIVE COUPLED-MODE THEORY}

As illustrated in Fig. 1, a circular Bragg microdisk resonator consists of a central disk of dielectric material surrounded by radial perturbation of Bragg gratings. The central disk region spans from the center to $\rho_{0}$ while the outer grating spans from $\rho_{0}$ to $\rho_{R}$. In the case that the polarization effects due to the waveguide structure are not concerned, we can start from the scalar Helmholtz equation in cylindrical coordinates

$$
\left[\frac{1}{\rho} \frac{\partial}{\partial \rho}\left(\rho \frac{\partial}{\partial \rho}\right)+\frac{1}{\rho^{2}} \frac{\partial^{2}}{\partial \varphi^{2}}+k_{0}^{2} n^{2}(\rho, z)+\frac{\partial^{2}}{\partial z^{2}}\right] E_{z}(\rho, \varphi, z)=0,
$$

*xksun@caltech.edu; http://www.its.caltech.edu/ aphyariv

Integrated Optics: Devices, Materials, and Technologies XII, edited by Christoph M. Greiner, Christoph A. Waechter Proc. of SPIE Vol. 6896, 689604, (2008) · 0277-786X/08/\$18 · doi: 10.1117/12.763948 
where $k_{0}=\frac{\omega}{c}=\frac{2 \pi}{\lambda_{0}}$ is the wave number in vacuum.

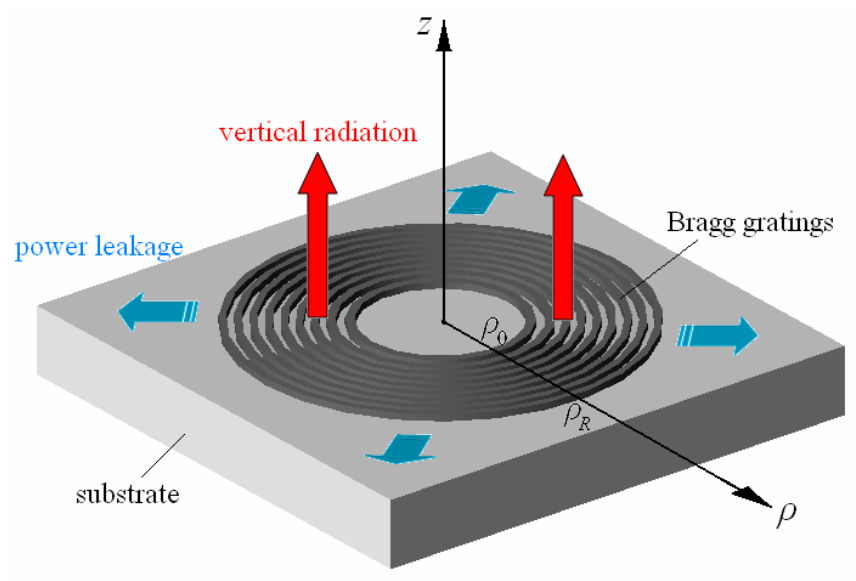

Fig. 1. Illustration of a circular Bragg microdisk laser.

For an azimuthally propagating eigenmode, $E_{z}$ in a passive uniform medium in which the dielectric constant $n^{2}(\rho, z)=\varepsilon_{r}(z)$ can be expressed as

$$
E_{z}(\rho, \varphi, z)=E_{z}^{(m)}(\rho, z) \exp (i m \varphi)=\left[A H_{m}^{(1)}(\beta \rho)+B H_{m}^{(2)}(\beta \rho)\right] Z(z) \exp (i m \varphi),
$$

where $m$ is the azimuthal mode number, $\beta=k_{0} n_{\text {eff }}$ is the in-plane propagation constant, and $Z(z)$ is the fundamental mode profile of the planar slab waveguide satisfying

$$
\left(k_{0}^{2} \varepsilon_{r}(z)+\frac{\partial^{2}}{\partial z^{2}}\right) Z(z)=\beta^{2} Z(z) .
$$

In a radially perturbed gain medium, the dielectric constant can be expressed as $n^{2}(\rho, z)=\varepsilon_{r}(z)+i \varepsilon_{i}(z)+\Delta \varepsilon(\rho, z)$ where $\left|\varepsilon_{i}(z)\right|<<\varepsilon_{r}(z)$ represents the gain/loss and $\Delta \varepsilon(\rho, z)$ reflects the contribution of perturbation. For optimal field confinement, the perturbation $\Delta \varepsilon(\rho, z)$ has to be expanded in Hankel-phased plane wave series ${ }^{1}$

$$
\begin{aligned}
& \Delta \varepsilon(\rho, z)=-\Delta \varepsilon_{0} \sum_{l= \pm 1, \pm 2} a_{l}(z) \exp \left(-i l \Phi\left[H_{m}^{(1)}\left(\beta_{\text {design }} \rho\right)\right]\right) \\
& =-\Delta \varepsilon_{0} \sum_{l= \pm 1, \pm 2} a_{l}(z) \exp \left(-i l \Phi\left[H_{m}^{(1)}(x)\right]\right) \exp (-i l \delta \cdot x) \\
& =-\Delta \varepsilon_{0}\left[a_{2}(z) \frac{H_{m}^{(2)}}{H_{m}^{(1)}} e^{-2 i \delta \cdot x}+a_{-2}(z) \frac{H_{m}^{(1)}}{H_{m}^{(2)}} e^{2 i \delta \cdot x}+a_{1}(z) \frac{H_{m}^{(2)}}{\left|H_{m}^{(1)}\right|} e^{-i \delta \cdot x}+a_{-1}(z) \frac{H_{m}^{(1)}}{\left|H_{m}^{(1)}\right|} e^{i \delta \cdot x}\right]
\end{aligned}
$$

In the above expression, $a_{l}(z)$ is the expansion coefficient of $\Delta \varepsilon(\rho, z)$ at a given $z . x$ is the normalized radius defined as $x=\beta \rho . \delta=\left(\beta_{\text {design }}-\beta\right) / \beta(|\delta|<<1)$, the normalized frequency detuning factor, represents the relative frequency shift from the optimal coupling design.

To account for the vertically radiating fields, we include an additional term $\Delta E(x, z)$ so that

$$
E_{z}^{(m)}(x, z)=\left[A(x) H_{m}^{(1)}(x)+B(x) H_{m}^{(2)}(x)\right] Z(z)+\Delta E(x, z) .
$$

Assuming that the radiating field $\Delta E(x, z)$ has an $\exp \left( \pm i k_{0} z\right)$ dependence on $z$ in free space, i.e.

$$
\left[\frac{1}{\rho} \frac{\partial}{\partial \rho}\left(\rho \frac{\partial}{\partial \rho}\right)-\frac{m^{2}}{\rho^{2}}\right] \Delta E=0,
$$


substituting (4), (5), (6) into (1), introducing the large-radius approximations ${ }^{1}$

$$
\left|\frac{H_{m}^{(1,2)}(x)}{x}\right| \ll\left|\frac{d H_{m}^{(1,2)}(x)}{d x}\right|, \frac{d^{n} H_{m}^{(1,2)}(x)}{d x^{n}} \approx( \pm i)^{n} H_{m}^{(1,2)}(x),
$$

neglecting the second derivatives of $A(x)$ and $B(x)$, and applying the modal solution in the passive unperturbed case, we find

$$
\begin{aligned}
& 2 i Z\left(\frac{d A}{d x} H_{m}^{(1)}-\frac{d B}{d x} H_{m}^{(2)}\right)+i \frac{k_{0}^{2} \varepsilon_{i}}{\beta^{2}}\left(A H_{m}^{(1)} Z+B H_{m}^{(2)} Z\right)+\frac{1}{\beta^{2}}\left(k_{0}^{2} \varepsilon_{r}+i k_{0}^{2} \varepsilon_{i}+\frac{\partial^{2}}{\partial z^{2}}\right) \Delta E \\
= & \frac{k_{0}^{2} \Delta \varepsilon_{0}}{\beta^{2}}\left[a_{2} \frac{H_{m}^{(2)}}{H_{m}^{(1)}} e^{-2 i \delta \cdot x}+a_{-2} \frac{H_{m}^{(1)}}{H_{m}^{(2)}} e^{2 i \delta \cdot x}+a_{1} \frac{H_{m}^{(2)}}{\left|H_{m}^{(1)}\right|} e^{-i \delta \cdot x}+a_{-1} \frac{H_{m}^{(1)}}{\left|H_{m}^{(1)}\right|} e^{i \delta \cdot x}\right]\left(A H_{m}^{(1)} Z+B H_{m}^{(2)} Z+\Delta E\right) .
\end{aligned}
$$

The phase-matching condition requires that the source and wave have close phase dependence. Grouping the terms with the same kind of Hankel functions leads to the following set of coupled equations

$$
\left\{\begin{array}{l}
2 i \frac{d A}{d x} H_{m}^{(1)} Z+i \frac{k_{0}^{2} \varepsilon_{i}}{\beta^{2}} A H_{m}^{(1)} Z=\frac{k_{0}^{2} \Delta \varepsilon_{0}}{\beta^{2}}\left(a_{-2} B H_{m}^{(1)} e^{2 i \delta \cdot x} Z+a_{-1} \frac{\Delta E}{\left|H_{m}^{(1)}\right|} H_{m}^{(1)} e^{i \delta \cdot x}\right) \\
-2 i \frac{d B}{d x} H_{m}^{(2)} Z+i \frac{k_{0}^{2} \varepsilon_{i}}{\beta^{2}} B H_{m}^{(2)} Z=\frac{k_{0}^{2} \Delta \varepsilon_{0}}{\beta^{2}}\left(a_{2} A H_{m}^{(2)} e^{-2 i \delta \cdot x} Z+a_{1} \frac{\Delta E}{\left|H_{m}^{(1)}\right|} H_{m}^{(2)} e^{-i \delta \cdot x}\right) \\
\left(k_{0}^{2} \varepsilon_{r}+\frac{\partial^{2}}{\partial z^{2}}\right) \Delta E=k_{0}^{2} \Delta \varepsilon_{0}\left(a_{1} A\left|H_{m}^{(1)}\right| e^{-i \delta \cdot x} Z+a_{-1} B\left|H_{m}^{(1)}\right| e^{i \delta \cdot x} Z\right)
\end{array}\right.
$$

From $(9 \mathrm{c}), \Delta E$ can be expressed as

$$
\Delta E=\left(s_{1} A e^{-i \delta \cdot x}+s_{-1} B e^{i \delta \cdot x}\right)\left|H_{m}^{(1)}\right|,
$$

where

$$
s_{l}(z)=k_{0}^{2} \Delta \varepsilon_{0} \int_{-\infty}^{+\infty} a_{l}\left(z^{\prime}\right) Z\left(z^{\prime}\right) G\left(z, z^{\prime}\right) d z^{\prime}
$$

and $G\left(z, z^{\prime}\right)$ is the Green's function satisfying $\left(k_{0}^{2} \varepsilon_{r}(z)+\frac{\partial^{2}}{\partial z^{2}}\right) G\left(z, z^{\prime}\right)=\delta\left(z-z^{\prime}\right)$.

Substituting (10) into (9a) and (9b), multiplying both sides by $Z(z)$ and integrating over $z$, we arrive at

$$
\left\{\begin{array}{l}
\frac{d A}{d x}=\left(g_{A}-h_{-1,1}\right) A-\left(h_{-1,-1}+i h_{-2}\right) B e^{2 i \delta \cdot x} \\
\frac{d B}{d x}=-\left(g_{A}-h_{1,-1}\right) B+\left(h_{1,1}+i h_{2}\right) A e^{-2 i \delta \cdot x}
\end{array}\right.
$$

where the gain coefficient $g_{A} \equiv-\frac{k_{0}^{2}}{2 P \beta^{2}} \int_{-\infty}^{+\infty} \varepsilon_{i}(z) Z^{2}(z) d z$, the radiation coupling coefficients $h_{ \pm 1, \pm 1}=\frac{i k_{0}^{2} \Delta \varepsilon_{0}}{2 P \beta^{2}} \int_{-\infty}^{+\infty} a_{ \pm 1}(z) s_{ \pm 1}(z) Z(z) d z$, the feedback coupling coefficients $h_{ \pm 2}=\frac{k_{0}^{2} \Delta \varepsilon_{0}}{2 P \beta^{2}} \int_{-\infty}^{+\infty} a_{ \pm 2}(z) Z^{2}(z) d z$, and the normalization constant $P \equiv \int_{-\infty}^{+\infty} Z^{2}(z) d z$.

In the case of index grating, we can choose the phase of the grating such that $a_{-1}=a_{1}, a_{-2}=a_{2}$, then we denote $h_{1}=h_{ \pm 1, \pm 1}$, $h_{2}=h_{ \pm 2}$. By defining $u=g_{A}-h_{1}$ and $v=h_{1}+i h_{2}$, the generic solution of (12) can be written as 


$$
\left\{\begin{array}{l}
A(x)=\left[C_{1} \exp (S x)+C_{2} \exp (-S x)\right] \exp (i \delta \cdot x) \\
B(x)=-\frac{1}{v}\left[C_{1}(S-u+i \delta) \exp (S x)-C_{2}(S+u-i \delta) \exp (-S x)\right] \exp (-i \delta \cdot x),
\end{array}\right.
$$

where $S \equiv \sqrt{(u-i \delta)^{2}-v^{2}}$. In analogy to the case of a linear grating ${ }^{4}$, the modes with a real $S$ manifest themselves as bandgap modes since they are located within the band gap in the band diagram and their fields are reflected by the grating. They are mostly confined in the guiding defect so that they are also termed as "defect modes." In the unperturbed region where $\Delta \varepsilon=0$, we have $h_{1}=h_{2}=0$, and (13) reduces to

$$
\left\{\begin{array}{l}
A(x)=A(0) \exp \left(g_{A} x\right) \\
B(x)=B(0) \exp \left(-g_{A} x\right)
\end{array}\right.
$$

\section{MODAL FIELD, CHARACTERISTIC EQUATION, AND OPTICAL LOSS FOR CIRCULAR BRAGG MICRODISK LASERS}

Referring to Fig. 1, to solve for the mode profile and characteristic equation for the microdisk lasers we invoke the following boundary conditions:

1. At the center $x=0$, the total amplitude of each Fourier component of the field must be finite, requiring $A(0)=B(0)=A$. Therefore, the amplitudes and the electric field in the disk region are given by

$$
\left\{\begin{array}{l}
A(x)=A \exp \left(g_{A} x\right) \\
B(x)=A \exp \left(-g_{A} x\right) \\
E_{z}^{(m)}(x, z)=A Z(z)\left(\exp \left(g_{A} x\right) H_{m}^{(1)}(x)+\exp \left(-g_{A} x\right) H_{m}^{(2)}(x)\right)
\end{array}\right.
$$

2. At the exterior boundary $x=x_{R}$, we assume there is no incoming wave from $x>x_{R}$, and therefore we have $B\left(x_{R}\right)=0$ which yields $C_{2}=\frac{S-u+i \delta}{S+u-i \delta} \exp \left(2 S x_{R}\right) C_{1}$. The amplitudes and the electric field in the grating region are then given by

$$
\left\{\begin{array}{l}
A(x)=C_{1} \exp (S x+i \delta \cdot x)\left[1+\frac{S-u+i \delta}{S+u-i \delta} \exp \left(2 S\left(x_{R}-x\right)\right)\right] \\
B(x)=-\frac{C_{1}(S-u+i \delta)}{v} \exp (S x-i \delta \cdot x)\left[1-\exp \left(2 S\left(x_{R}-x\right)\right)\right] \\
E_{z}^{(m)}(x, z)=C_{1} \exp (S x+i \delta \cdot x)\left[1+\frac{S-u+i \delta}{S+u-i \delta} \exp \left(2 S\left(x_{R}-x\right)\right)\right]\left(H_{m}^{(1)}(x) Z(z)+S_{1}(z) \exp (-i \delta \cdot x)\left|H_{m}^{(1)}(x)\right|\right) \\
\quad-\frac{C_{1}(S-u+i \delta)}{v} \exp (S x-i \delta \cdot x)\left[1-\exp \left(2 S\left(x_{R}-x\right)\right)\right]\left(H_{m}^{(2)}(x) Z(z)+S_{-1}(z) \exp (i \delta \cdot x)\left|H_{m}^{(1)}(x)\right|\right)
\end{array}\right.
$$

3. At the interface $x=x_{0}$, both the field and its first order derivative must be continuous (for the TE mode). After imposing these two restrictions we end up with the characteristic equation for the circular Bragg microdisk lasers

$$
\begin{aligned}
& \frac{\exp \left(2 g_{A} x_{0}\right) H_{m}^{(1)}\left(x_{0}\right)+H_{m}^{(2)}\left(x_{0}\right)}{\left(g_{A}+i\right)\left[\exp \left(2 g_{A} x_{0}\right) H_{m}^{(1)}\left(x_{0}\right)-H_{m}^{(2)}\left(x_{0}\right)\right]} \\
= & \frac{\left[1+\frac{S-u+i \delta}{S+u-i \delta} \exp \left(2 S\left(x_{R}-x_{0}\right)\right)\right] \exp \left(2 i \delta \cdot x_{0}\right) H_{m}^{(1)}\left(x_{0}\right)-\frac{(S-u+i \delta)}{v}\left[1-\exp \left(2 S\left(x_{R}-x_{0}\right)\right)\right] H_{m}^{(2)}\left(x_{0}\right)}{\left\{\begin{array}{l}
{\left[S+i \delta-(S-i \delta) \frac{S-u+i \delta}{S+u-i \delta} \exp \left(2 S\left(x_{R}-x_{0}\right)\right)\right] \exp \left(2 i \delta \cdot x_{0}\right) H_{m}^{(1)}\left(x_{0}\right)+i\left[1+\frac{S-u+i \delta}{S+u-i \delta} \exp \left(2 S\left(x_{R}-x_{0}\right)\right)\right] \exp \left(2 i \delta \cdot x_{0}\right) H_{m}^{(1)}\left(x_{0}\right)} \\
-\frac{(S-u+i \delta)}{v}\left[S-i \delta+(S+i \delta) \exp \left(2 S\left(x_{R}-x_{0}\right)\right)\right] H_{m}^{(2)}\left(x_{0}\right)+i \frac{(S-u+i \delta)}{v}\left[1-\exp \left(2 S\left(x_{R}-x_{0}\right)\right)\right] H_{m}^{(2)}\left(x_{0}\right)
\end{array}\right\}}
\end{aligned}
$$


during which we have used $\int_{-\infty}^{+\infty} s_{ \pm 1}(z) Z(z) d z=0$. Eq. (17) is a complex equation, whose solution yields the threshold gain $g_{A}$ and the frequency detuning $\delta$ simultaneously.

As indicated in Fig. 1, the power loss in a circular Bragg microdisk laser consists of two contributions: the coherently scattered, vertically emitted light (our useful signal) due to the first-order Bragg diffraction, and the transverse loss due to the finite radial length of the Bragg reflector. Using the total energy stored in the resonator $\frac{1}{4} \int_{V}\left(\varepsilon|\mathbf{E}|^{2}+\mu|\mathbf{H}|^{2}\right) d V$, and the outflow power through a surface $\int_{S}(\mathbf{E} \times \mathbf{H}) \cdot d \mathbf{A}$, we define the normalized total optical loss $L$ as

$$
L=\frac{\int_{0}^{2 \pi} \int_{\rho_{0}}^{\rho_{R}}|\Delta E(\rho, z=0)|^{2} \rho d \rho d \varphi}{\int_{0}^{D} \int_{0}^{2 \pi} \int_{0}^{\rho_{R}}|E(\rho, z)|^{2} \rho d \rho d \varphi d z}+\frac{\int_{0}^{D} \int_{0}^{2 \pi}\left|E\left(\rho=\rho_{R}, z\right)\right|^{2} \rho_{R} d \varphi d z}{\int_{0}^{D} \int_{0}^{2 \pi} \int_{0}^{\rho_{R}}|E(\rho, z)|^{2} \rho d \rho d \varphi d z}
$$

where $D$ is the thickness (vertical dimension) of the resonator.

It should be noted that, in the calculation of the threshold gain levels we consider only the loss stemming from finite Bragg reflectivity and the vertical radiation. Other loss mechanisms, such as free carrier absorption or random scattering, are not included in our model.

\section{OPTIMAL DESIGN PROCEDURE FOR CIRCULAR BRAGG MICRODISK LASERS}

\subsection{Description of the example for numerical study}

Since we have previously fabricated such circular Bragg lasers with chirped grating in InGaAsP active semiconductor material ${ }^{5}$, we use the layer structure therein as an example for our numerical study. The target lasing wavelength $\lambda_{0}$ is $1.55 \mu \mathrm{m}$. For simplicity, we approximate the complicated layer structure by an effective index profile comprising five layers as illustrated in Table 1. Numerical calculations of the mode profile and the effective index of the approximated layer structure indicate negligible deviations from those of the exact one. We focus our analysis on the case of shallow gratings with an etch depth of $185 \mathrm{~nm}$. The vertical mode profile $Z(z)$ and the effective index $n_{\text {eff }}$ are calculated by a 1-D mode solver (with a Transverse Matrix Method). The Green's function is also numerically calculated and displayed in Fig. 2 for $-\infty<z<\infty, z^{\prime}$ between the top and bottom surfaces of the resonator.

Table. 2. Approximated layer structure for numerical study.

\begin{tabular}{|l|l|l|}
\hline Layer description & Refractive index $\mathrm{n}$ & Thickness \\
\hline Upper cladding & 1.54 & $\infty$ \\
\hline Third layer & 3.281 & $60.5 \mathrm{~nm}$ \\
\hline Second layer (active region) & 3.4057 & $129 \mathrm{~nm}$ \\
\hline First layer & 3.281 & $60.5 \mathrm{~nm}$ \\
\hline Lower cladding & 1.54 & $\infty$ \\
\hline
\end{tabular}




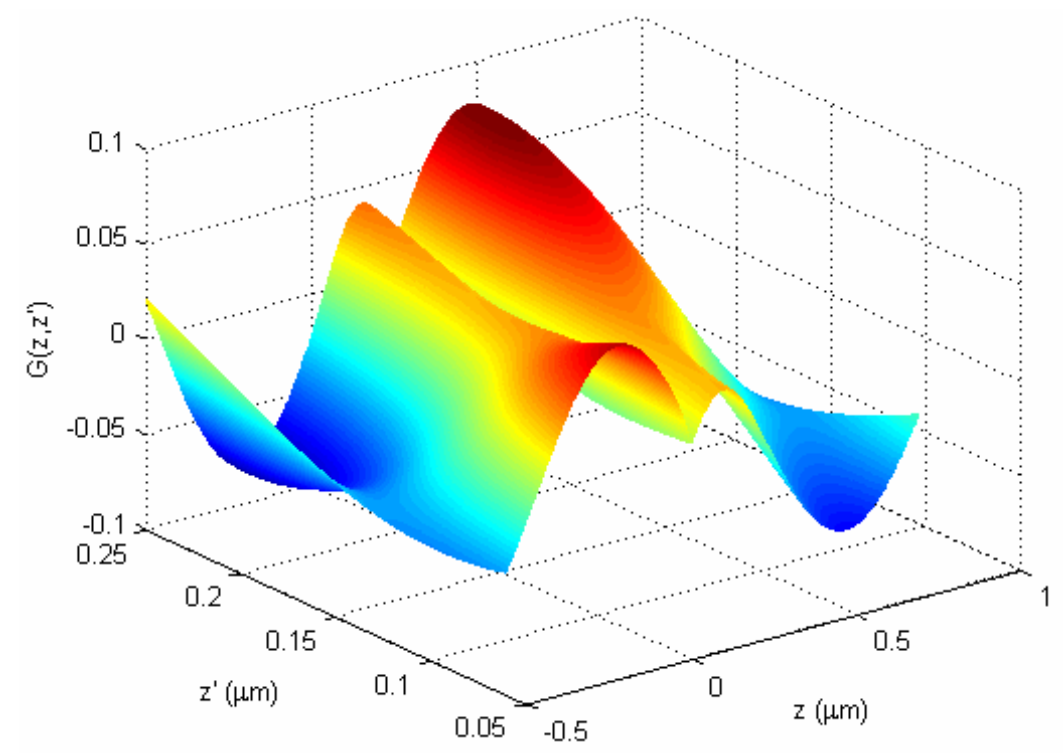

Fig. 2. Real part of the Green's function $G\left(z, z^{\prime}\right)$ with $-\infty<z<\infty, z^{\prime}$ between the top and bottom surfaces of the resonator.

\subsection{Optimal design procedure}

In view of current fabrication process, we prefer a rectangular grating profile with a Hankel-phased modulation ${ }^{1}$

$$
\begin{aligned}
\Theta\left(\Phi\left[H_{m}^{(1)}(x)\right], \alpha\right)=\left\{\begin{array}{ll}
1, & \cos \left(\Phi\left[H_{m}^{(1)}(x)\right]\right) \geq \alpha \\
0, & \cos \left(\Phi\left[H_{m}^{(1)}(x)\right]\right)<\alpha
\end{array},\right. \text { which can be expanded in Fourier series as } \\
\quad \frac{\arccos \alpha}{\pi}+\frac{2}{\pi} \sum_{l=1}^{\infty} \frac{\sin (l \arccos \alpha)}{l} \cos \left(l \Phi\left[H_{m}^{(1)}(x)\right]\right) \\
\approx \frac{\arccos \alpha}{\pi}+\frac{2}{\pi}\left[\sin (\arccos \alpha) \cos \left(\Phi\left[H_{m}^{(1)}(x)\right]\right)+\frac{\sin (2 \arccos \alpha)}{2} \cos \left(2 \Phi\left[H_{m}^{(1)}(x)\right]\right)\right] \\
=d_{c}+\frac{1}{2 \pi} \sin \left(2 \pi d_{c}\right)\left[\exp \left(-i 2 \Phi\left[H_{m}^{(1)}(x)\right]\right)+\exp \left(i 2 \Phi\left[H_{m}^{(1)}(x)\right]\right)\right]+\frac{1}{\pi} \sin \left(\pi d_{c}\right)\left[\exp \left(-i \Phi\left[H_{m}^{(1)}(x)\right]\right)+\exp \left(i \Phi\left[H_{m}^{(1)}(x)\right]\right)\right]
\end{aligned}
$$

So the expansion yields the coefficients $a_{2}=a_{-2}=\frac{\sin \left(2 \pi d_{c}\right)}{2 \pi}$ and $a_{1}=a_{-1}=\frac{\sin \left(\pi d_{c}\right)}{\pi}$ where $d_{c} \equiv \frac{\arccos \alpha}{\pi}(-1<\alpha<1$, $\left.0<d_{c}<1\right)$ is the duty cycle of the Hankel-phased rectangular grating. It should be emphasized that the duty cycle has a significant role in determining the coupling coefficients $h_{1}$ and $h_{2}^{6}$. A judicious choice is $d_{c}=0.25$ where $h_{2}$ is maximal and $\operatorname{Re}\left(h_{1}\right)$ is not small so that we expect a large ratio of useful signal to power leakage, hence high emission efficiency. In such a condition, the coefficients are found to be $h_{1}=0.0072+0.0108 i$ and $h_{2}=0.0601$.

It is well-known that larger devices, with more Bragg layers, usually have lower threshold levels due to more efficient light confinement. Therefore, we predefine the overall size of the device, keeping the exterior boundary $x_{R}$ fixed and optimize the inner radius $x_{0}$. We assume a typical device with a diameter of $\sim 20 \mu \mathrm{m}\left(\rho_{R} \approx 10 \mu \mathrm{m}\right)$, so $x_{R}=\beta \rho_{R}=2 \pi / \lambda_{0} \times n_{e f f} \times \rho_{R}$ $\approx 2 \pi / 1.55 \times 2.83 \times 10 \approx 120$. Given a pair of $x_{0}$ and $x_{R}$, we put them into Eq. (17), solve for all the allowed pairs of $g_{A}$ and $\delta$, then pick up amongst them the mode which has the smallest $g_{A}$ (the first lasing mode). Fig. 3 shows the threshold gain $g_{A}$ and the frequency detuning factor $\delta$ of the first lasing mode as a function of $x_{0}$. Fig. 4 depicts the normalized total optical loss $L$ and the emission efficiency $\eta$. Since the lasing threshold is directly related to the total loss of a resonator in our model, it's intuitively pleasing to see, by a comparison of Figs. 3 and 4, that the threshold gain $g_{A}$ and the total optical loss $L$, though obtained in different ways, have a highly similar dependence on $x_{0}$. We note that for $x_{0}$ between 25 and 90 , the threshold gain remains very low, which indicates that in this regime the resonator has a relatively high $Q$ value. Also Fig. 4 shows a peak of the emission efficiency at $x_{0}=45$. The numerical results give that for $x_{0}=45$ the threshold gain $g_{A}$ of the first lasing mode is $4.16 \times 10^{-4}$, which is within the low threshold regime, thus making it the desired (optimal) 
design. Actually, the highest emission efficiency at the optimal point $x_{0}=45$ is mainly due to the minimal power leakage at that point, as confirmed in Fig. 5, which displays the normalized radial profiles of the in-plane electric fields for designs of different disk radii $x_{0}$.

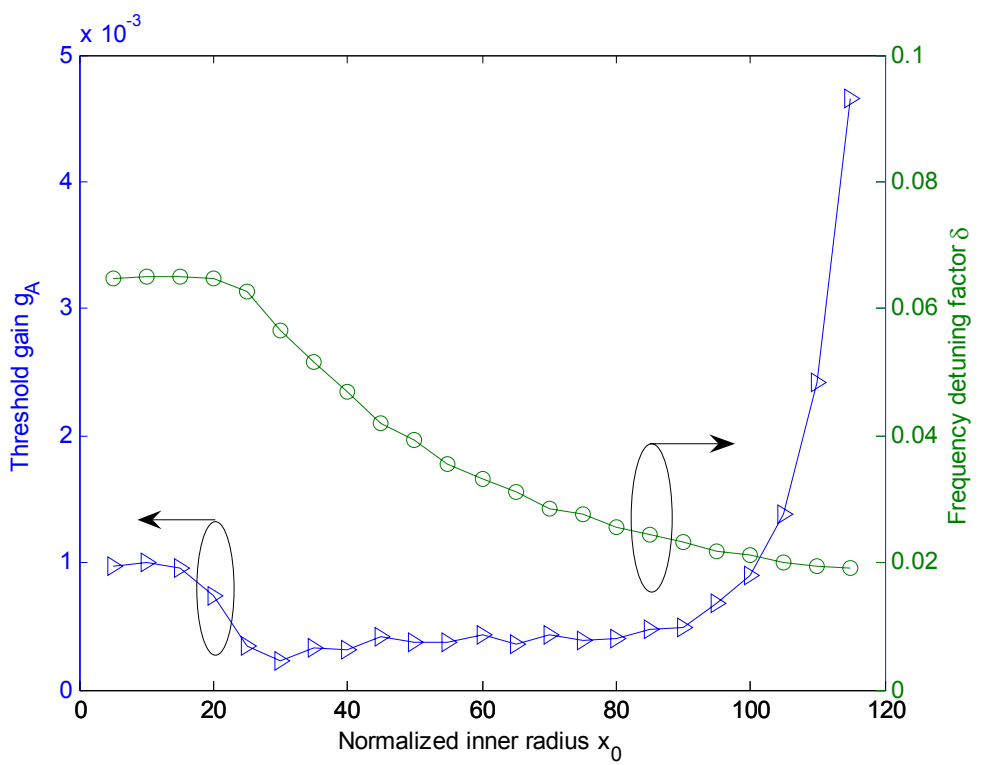

Fig. 3. Threshold gain $g_{A}$ and frequency detuning factor $\delta$ of the first lasing modes of the circular Bragg microdisk lasers with different inner radii $x_{0}$.

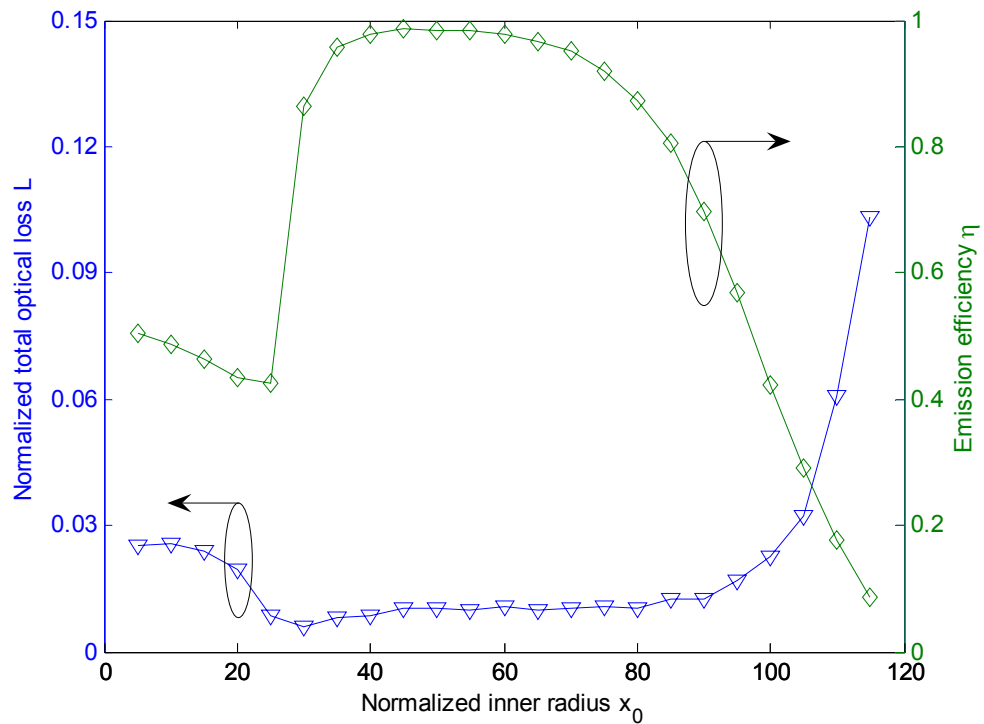

Fig. 4. Normalized total optical loss and emission efficiency of the first lasing modes of the circular Bragg microdisk lasers with different inner radii $x_{0}$. 


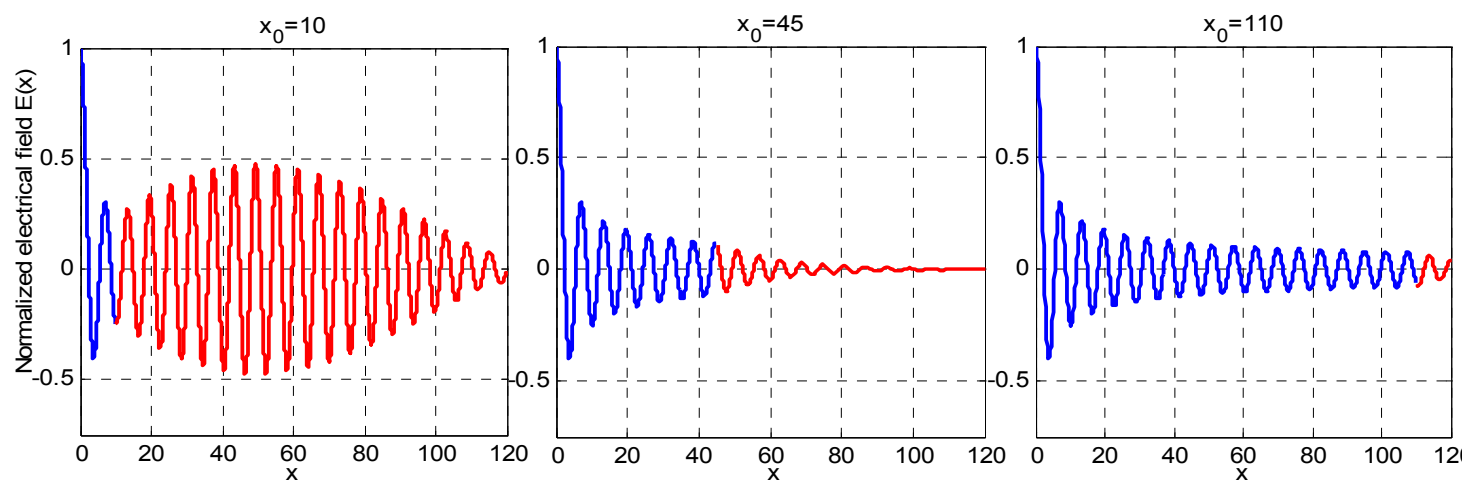

Fig. 5. Normalized radial profiles of the in-plane electric fields $E(x)$ for designs with $x_{0}=10,45$, and 110 . The fields in the inner disk and in the outer grating are marked with blue and red, respectively.

\subsection{Discussion}

Seen from Figs. 3 and 4, the threshold gain $g_{A}$ and the total optical loss $L$ remain low for $25 \leq x_{0} \leq 90$ but increase rapidly for smaller and larger $x_{0}$. Similar trends were found for devices employing different duty cycles. These trends stem from the presetting of the overall size of the resonator $x_{R}$. For large $x_{0}$, there are fewer Bragg gratings in the external reflector which, consequently, reduces its reflectivity resulting in more power leakage. On the other hand, shorter grating region also reduces the emission from the surface, thus creating a tradeoff between the two sources of loss - vertical radiation and periphery power leakage. This tradeoff results in an optimal $x_{0}$ which minimizes the total optical loss and the threshold gain level.

\section{REFERENCES}

J. Scheuer and A. Yariv, "Coupled-Waves Approach to the Design and Analysis of Bragg and Photonic Crystal Annual Resonators," IEEE J. Quantum Electron. 39 (12), 1555-1562 (2003).

2 J. Scheuer and A. Yariv, "Annular Bragg defect mode resonators," J. Opt. Soc. Am. B 20 (11), 2285-2291 (2003); J. Scheuer, W. M. J. Green, G. DeRose et al., "Low-threshold two-dimensional annular Bragg lasers," Opt. Lett. 29 (22), 2641-2643 (2004).

3 J. Scheuer, W. M. J. Green, G. A. DeRose et al., "InGaAsP Annular Bragg Lasers: Theory, Applications, and Modal Properties," IEEE J. Sel. Top. Quantum Electron. 11 (2), 476-484 (2005).

4 Amnon Yariv, Optical Electronics in Modern Communications, 5th ed. (Oxford Univ. Press, New York, 1997).

5 W. M. J. Green, J. Scheuer, G. DeRose et al., "Vertically emitting annular Bragg lasers using polymer epitaxial transfer," Appl. Phys. Lett. 85 (17), 3669-3671 (2004).

6 G. F. Barlow, A. Shore, G. A. Turnbull et al., "Design and analysis of a low-threshold polymer circular-grating distributed-feedback laser," J. Opt. Soc. Am. B 21 (12), 2142-2150 (2004). 